CLINICAL STUDY

\title{
Bone mineral density in male adolescents with autism spectrum disorders and disruptive behavior disorder with or without antipsychotic treatment
}

\author{
Yvette Roke $^{1}$, Peter N van Harten ${ }^{1,2}$, Jan K Buitelaar ${ }^{3,4}$, Diederik E Tenback ${ }^{1,5}$, Lorentz G B A Quekel ${ }^{6}$, \\ Yolanda B de Rijke ${ }^{7}$ and Annemieke M Boot ${ }^{8}$ \\ ${ }^{1}$ GGz Central Psychiatric Centre, PO Box 3051, 3800 DB Amersfoort, The Netherlands, ${ }^{2}$ The University of Maastricht, Maastricht, The Netherlands, \\ ${ }^{3}$ Department of Cognitive Neuroscience, Radboud University Nijmegen Medical Centre, Nijmegen, The Netherlands, ${ }^{4}$ Karakter Child and Adolescent \\ Psychiatry University Centre, Nijmegen, The Netherlands, ${ }^{5}$ The University of Utrecht, Utrecht, The Netherlands, ${ }^{6}$ Department of Radiology, Meander \\ Medical Center, Amersfoort, The Netherlands, ${ }^{7}$ Departments of Clinical Chemistry and Endocrine Laboratory, Erasmus Medical Centre, Rotterdam, The \\ Netherlands and ${ }^{8}$ Division of Paediatric Endocrinology, Department of Paediatrics, University Medical Centre Groningen, Groningen, The Netherlands \\ (Correspondence should be addressed to Y Roke; Email: y.roke@ggzcentraal.nl)
}

\begin{abstract}
Objective: To investigate the long-term effects of antipsychotic (AP) treatment and AP-induced hyperprolactinemia on bone mineral density (BMD) and body composition in male adolescents with autism spectrum disorders (ASDs) and/or disruptive behavior disorder (DBD).

Design: Physically healthy 10- to 20-year-old boys with ASD and/or DBD, chronically treated $(n=56$; mean 52 months, range 16-126 months) or not treated $(n=47)$ with an AP, were recruited to this observational study. Prolactin levels and biochemical bone parameters were measured and BMD of the lumbar spine and total body, and body composition were assessed by dual-energy X-ray absorptiometry, and volumetric BMD of the lumbar spine calculated. Group differences were tested with Student's $t$-test, $\chi^{2}$ test, Fisher exact test, and logistic regression analysis.

Results: Forty-nine percent of the boys treated with an AP had hyperprolactinemia. The mean volumetric lumbar spine BMD $z$-score was lower $(P=0.043)$, the total percentage of body fat $z$-score was higher $(P=0.042)$, and biochemical bone marker carboxyterminal cross-linking telopeptide of bone collagen was lower in the AP-treated boys with hyperprolactinemia than in the AP-treated boys without hyperprolactinemia. Seven to $11 \%$ of the hyperprolactinemic boys had low BMD. The mean lumbar spine and total body BMD z-scores and body composition were similar in the boys who were or were not treated with an AP. The total study population had a lower mean lean tissue mass (mean $z$-score $-0.37, P=0.004$ ) and a higher percentage of total body fat (mean $z$-score $1.16, P<0.001$ ) than healthy controls (normative data); biochemical bone parameters were within normal limits. Conclusion: AP-induced hyperprolactinemia in boys with ASD or DBD may have a negative effect on lumbar spine BMD. Longitudinal studies are needed to confirm this finding and further disentangle the effects of the disorder, lifestyle, treatment, and hyperprolactinemia.
\end{abstract}

European Journal of Endocrinology 167 855-863

\section{Introduction}

Children and adolescents with autism spectrum disorders (ASDs) are often treated with antipsychotic (AP) medication, mostly second-generation AP because the incidence of extrapyramidal side effects is lower than with first-generation AP (1). AP reduce maladaptive behaviors, such as irritability, self-injury, stereotypies, and hyperactivity, in children and adolescents with ASD, and reduce irritability and aggressive behavior in children and adolescents with disruptive behavior disorder (DBD) (2).

While the Food and Drug Administration (FDA) in the USA has approved risperidone and aripiprazole for the treatment of children and adolescents with irritability associated with $\operatorname{ASD}(3,4,5)$, these drugs have not yet been approved for this indication in Europe. AP are also frequently used, off-label, to treat severe aggression in children and adolescents with DBD. Despite their efficacy (5), there are concerns about metabolic (such as obesity, hyperglycemia, and dyslipidemia) and endocrine (such as hyperprolactinemia) side effects $(6,7,8)$. AP such as olanzapine, clozapine, and risperidone can reduce hepatic insulin sensitivity (9), possibly by reducing serotonin levels in the brain, which causes a reduction in osteocalcin and adiponectin levels and ultimately diminished insulin sensitivity (10). AP-linked obesity and type 2 diabetes mellitus are more prevalent in children and adolescents than in adults (7), possibly because of differences in their body 
composition, e.g. a higher percentage of body fat and less lean body mass. Adolescence is the most important time in terms of attaining peak bone mass (11), and an increase in lean body mass, mainly muscles, is important for optimal bone mass (12). Disturbances in this process during puberty may increase the risk of osteoporosis later in life. The AP-induced change in energy metabolism and insulin signaling could lead to a lower bone mineral density (BMD) (10), which in turn could increase the risk of osteoporosis later in life.

AP-induced hyperprolactinemia could influence bone metabolism in two ways. First, hyperprolactinemia might directly affect bone turnover by stimulating bone resorption relative to bone formation $(13,14)$. Second, prolonged hyperprolactinemia may cause hypogonadotropic hypogonadism (15), resulting in suppression of GNRH secretion in the hypothalamus and diminished secretion of LH and FSH by the pituitary gland, resulting in a diminished secretion of sex hormones and ultimately in changes in bone metabolism. Patients with psychiatric disorders also have other risk factors for osteopenia, such as smoking, low calcium intake, vitamin D deficiency, and insufficient exercise.

Only one study has investigated the effect of AP-induced hyperprolactinemia on BMD in boys with different diagnoses $(n=83$; mean age 11.9 years, S.D. 2.8$)$ (16). BMD was lower in boys with hyperprolactinemia than in boys without hyperprolactinemia, but comparisons with healthy controls were not made. In adolescents with prolactinomas, in which prolactin levels are at least two to four times higher than in AP-induced hyperprolactinemia, BMD was decreased and was not restored after 2 years of treatment with dopamine agonists (17). Several studies of adults have shown AP-induced hypogonadism to be associated with a decreased BMD $(18,19,20,21,22)$ and two large case-control studies (44 500 patients and 16341 patients) showed AP medication to be associated with a twofold increased risk of hip or femur fractures $(23,24)$.

In this study, we investigated the consequences of long-term AP treatment and AP-induced hyperprolactinemia on BMD, biochemical bone parameters, and body composition in boys with ASD and/or DBD compared with boys with similar disorders never treated with AP.

\section{Materials and methods}

\section{Subjects}

All participants were recruited from the patient population of the adolescent outpatient clinic of the GGz Central Psychiatric Center, The Netherlands, from October 2006 to November 2009. Eligible participants were aged between 10 and 20 years, diagnosed with a psychiatric disorder, treated with potential prolactinelevating AP medication continuously for more than 16 months, and had an IQ above 85 . All participants
( 56 boys, 6 girls; $80 \%$ of the eligible population) had a diagnosis of ASD or DBD and were treated primarily with risperidone (57 out of 62). Comparison subjects ( 47 boys and 10 girls; $50 \%$ of the eligible population) met the same inclusion criteria except that they had never been treated with AP. The main reason for not participating was fear of venipuncture. None of the participants had a history of chronic diseases affecting puberty, endocrine disorders, thyroid disorders, bone diseases, use of oral corticosteroid or anticonvulsants, or known causes of hyperprolactinemia (25). The data for the girls (16 in a total study sample of 119) were not analyzed because of the low number of girls. Thus, the AP group consisted of 56 boys with ASD or DBD treated with AP and the comparison group consisted of 47 boys with ASD or DBD who had never been treated with AP. Use of other concomitant medication was permitted (Table 1). The first author contacted the parents/legal guardians and the patients by telephone and letter. The Local Medical Ethics Committee approved the study and written informed consent was obtained from the patient and their parents/legal guardians after the study procedures had been clearly explained.

\section{Methods}

The clinical diagnosis was obtained from the patients' chart. The first author measured the participants' height (without shoes, to the nearest $0.1 \mathrm{~cm}$ using a stadiometer, Seca 206, The Netherlands) and weight (participant wearing underwear, to the nearest $0.1 \mathrm{~kg}$, using an analog scale, Seca 761, The Netherlands). Age-specific height, weight, and BMI z-scores were calculated using normative data for male Dutch children $(26,27)$. Pubertal stage was established by showing the boys different Tanner stage photographs of the genitals ( $\mathrm{G}$ stage) and pubic hair ( $\mathrm{P}$ stage) and asking them to select the photographs that most accurately reflected their appearance (28). One of the parents/legal guardians was present during the selfassessment and confirmed the stages pointed out. The severity of disease was measured using the Clinical Global Impression-Severity scale (29). It is a 7-point scale that requires the first author to rate the severity of the patient's illness at the time of assessment, relative to the past experience with patients who have the same diagnosis. Considering total clinical experience, a patient is assessed on severity of mental illness at the time of rating: 1 , normal, not at all ill; 2 , borderline mentally ill; 3, mildly ill; 4, moderately ill; 5, markedly ill; 6, severely ill; or 7, extremely ill.

The average calcium intake (mg/day) was determined using the food frequency questionnaire for dairy products (30). Physical activity was divided into leisure sports, activity at school/work, home activities, and transport (biking or walking) (31). Each activity was expressed in terms of its ground reaction force (GRF) $(32,33)$ and then classified into four categories: 0, GRF 
Table 1 Characteristics of adolescents with autism spectrum disorders who were (cases, $n=56$ ) or who were not (comparison subjects, $n=47$ ) treated with antipsychotics.

\begin{tabular}{|c|c|c|c|}
\hline & $\begin{array}{l}\text { Cases }(n=56) \\
\text { Mean (S.D., range) }\end{array}$ & $\begin{array}{c}\text { Comparison subjects }(n=47) \\
\text { Mean (S.D., range) }\end{array}$ & $P$ value \\
\hline Age (years) & $14.8(2.2 ; 10,19)$ & $15.0(1.6 ; 12,18)$ & $0.50^{\mathrm{a}}$ \\
\hline Height $z$-score $(\mathrm{cm})$ & $0.43(1.1 ;-2.4,2.12)$ & $0.36(0.94 ;-1.5,2.9)$ & $0.37^{\mathrm{a}}$ \\
\hline BMI $z$-score & $0.25(1 ;-1.8,2.8)$ & $-0.08(1.2 ;-3.3,2.3)$ & $0.15^{\mathrm{a}}$ \\
\hline Tanner stage I/II/III/IV/V (n) & $0 / 8 / 16 / 22 / 10$ & $0 / 9 / 13 / 19 / 6$ & \\
\hline Caucasian ethnicity $(n(\%))$ & $55(98)$ & $46(96)$ & $0.54^{b}$ \\
\hline \multicolumn{4}{|l|}{ Primary diagnosis $(n(\%))$} \\
\hline ASD & $52(93)$ & $40(85)$ & $0.17^{\mathrm{b}}$ \\
\hline DBD & $4(7)$ & $7(15)$ & \\
\hline CGI-S & $4.7(1 ; 1-7)$ & $4.0(0.6 ; 3-6)$ & $0.003^{a}$ \\
\hline Antipsychotic dose (mg/day) & $1.6(0.8 ; 0.5,4)$ & & \\
\hline Duration of use (months) & $51.4(28 ; 16-126)$ & & \\
\hline \multicolumn{4}{|l|}{ Concomitant medication $(n(\%))$} \\
\hline Melatonin & $5(9)$ & $4(9)$ & \\
\hline SSRI & $2(4)$ & 0 & \\
\hline Psychostimulants & $10(18)$ & $13(28)$ & \\
\hline Atomoxetine & $2(4)$ & 0 & \\
\hline Calcium intake (mg/day) & $1314(725 ; 268,4164)$ & $992(564 ; 5-2958)$ & $0.008^{\mathrm{C}}$ \\
\hline GRF & $18(7 ; 3,40)$ & $19(6 ; 10,34)$ & $0.25^{\mathrm{c}}$ \\
\hline History of fracture(s; $n(\%))$ & $10(18)$ & $12(26)$ & $0.34^{\mathrm{b}}$ \\
\hline 25-Hydroxyvitamin D ( $\mathrm{nmol} / \mathrm{l})$ & $55.3(22 ; 14,107)$ & $66.4(23 ; 14,120)$ & $0.017^{\mathrm{a}}$ \\
\hline Deficient $(n(\%))$ & $8(14)$ & $5(11)$ & 0.77 \\
\hline Insufficient $(n(\%))$ & $24(43)$ & $13(28)$ & 0.15 \\
\hline
\end{tabular}

ASD, autism spectrum disorders; DBD, disruptive behavior disorder; GRF, ground reaction force; SSRI, selective serotonin reuptake inhibitor; CGI-S, the Clinical Global Impression-Severity scale; history of fracture(s), number of patients that ever had one or more fractures from birth until now.

andependent sample Student's $t$-test.

${ }^{\mathrm{b}} \chi^{2}$ test.

'Mann-Whitney $U$ test.

$<1 \times$ body weight, e.g. swimming, cycling; 1, GRF between 1 and $2 \times$ body weight, e.g. rowing, dancing; 2 , GRF between 2 and $4 \times$ body weight, e.g. running, hockey, soccer; 3, GRF $>4 \times$ body weight, e.g. basketball, volleyball. The GRF score reported is the sum of the GRF scores.

A non-fasting morning blood sample was collected at about $0930 \mathrm{~h}$; a light breakfast had been eaten at about $0730 \mathrm{~h}$. Serum prolactin was measured with an automated chemiluminescence assay system (Immulite 2000, Siemens Healthcare Diagnostics, Beda, Brabant, The Netherlands) calibrated on the third IS 84/500. As macroprolactin, a complex of prolactin and $\operatorname{IgG}$ without biological activity, is responsible for $10 \%$ of cases of hyperprolactinemia (34), seven samples with prolactin concentrations higher than $37 \mathrm{ng} / \mathrm{ml}$ were assessed for the presence of macroprolactin. Polyethylene glycol $6000,200 \mu \mathrm{l},(25 \% \mathrm{w} / \mathrm{w})$ was added to $200 \mu \mathrm{l}$ serum and, after thorough vortex mixing, the mixture was centrifuged at $2200 \boldsymbol{g}$ for $30 \mathrm{~min}$ at $4{ }^{\circ} \mathrm{C}$. Subsequently, prolactin was measured in the supernatant and the untreated serum. The recovery of prolactin after precipitation with polyethylene glycol 6000 was $101.4 \pm 9.6 \%$ (S.D.), which excluded the presence of macroprolactin. TSH was measured by means of a Vitros Eci System (Ortho Diagnostics, Raritan, NJ, USA) to exclude children or adolescents with hyperprolactinemia due to thyroid disorders. Hyperprolactinemia was defined as a prolactin level above the 97.5th percentile based on normative data for age and sex (35).
Various biochemical bone parameters were measured. Type 1 amino-terminal propeptide of procollagen (P1NP) and bone-specific alkaline phosphatase (BAP) were measured as markers of bone formation and carboxyterminal cross-linking telopeptide of bone collagen (CTx) as marker of bone resorption. Calcium was measured with the Modular E170 (Roche Diagnostics) and BAP was measured by an immunoenzymetric assay using the Ostase BAP kit (IDS, Frankfurt am Main, Germany) with an inter-assay coefficient of variation (CV) of $<7.5 \%$. 25-Hydroxyvitamin $\mathrm{D}_{3}$ was assessed by RIA (DiaSorin, Inc., Saluggia, Vercelli, Italy) with intraassay and interassay $\mathrm{CV}$ of $<7$ and $<12 \%$ respectively. P1NP was also measured by RIA (Orion Diagnostica Oy, Espoo, Finland) with intra-assay and interassay CV of 13.7 and $<6.4 \%$ respectively. CTx was measured in serum by sandwich electrochemiluminescence immunoassay (Roche Diagnostics); the interassay CV was $1 \%$. P1NP, BAP, and CTx, were compared with literature reference values for age and sex $(36,37)$. 25-Hydroxyvitamin $\mathrm{D}_{3}$ levels below $30 \mathrm{nmol} / \mathrm{l}$ were considered deficient and below $50 \mathrm{nmol} / \mathrm{l}$ as insufficient (38).

BMD $\left(\mathrm{g} / \mathrm{cm}^{2}\right)$ of the lumbar spine and total body was measured by dual-energy X-ray absorptiometry (DXA, Lunar Prodigy). The reported CV is $1.0 \%$ for lumbar spine BMD and $0.73 \%$ for total body BMD (39). Quality assurance was performed daily. Ancillary DXA-derived data were used to calculate lumbar spine volumetric $\mathrm{BMD}$, to adjust for bone size, bone mineral apparent 
density (BMAD), with the model $\mathrm{BMAD}=\mathrm{BMD} \times$ $(4 /(\pi \times$ width $)(36,40,41)$.

Body composition was measured as bone mineral content (BMC), lean tissue mass (lean, in grams), and fat mass (DXA, Lunar Prodigy), and total percentage of body fat was determined. The $\mathrm{CV}$ for these measurements is reported as $1.29 \%$ for lean tissue mass, $2.59 \%$ for fat mass, and $0.74 \%$ for BMC (39). In the analysis, lean body mass and total percentage of body fat were used. The results were compared with age- and sex-matched Dutch reference values $(36,41)$ and expressed as z-scores. Plasma levels of risperidone, 9-hydroxyrisperidone, and pimozide were measured to evaluate compliance.

\section{Statistical analysis}

One-sample $t$-tests were used to compare the mean $z$-scores of the boys with age-matched normative data. To test for differences between the two study groups, Student's $t$-test, $\chi^{2}$ test, Mann-Whitney, or Fisher exact test was applied according to the type and distribution of the data. Dose equivalence was assumed between pimozide $(n=3)$ and risperidone $(n=53)(42,43)$.

Multiple linear regression was used to investigate whether BMD, body composition, and biochemical bone parameters were different between the two groups, with adjustment for BMI $z$-score, average daily calcium intake, and serum 25-hydroxyvitamin D3 level. Low BMD was defined as a $z$-score $\leq-2$ (44). Pearson's correlation coefficient was calculated to investigate the relationship between prolactin levels and the biochemical bone markers. Both hyperprolactinemia and inactivity are associated with weight gain, and therefore, we also investigated the relationship between prolactin and GRF score and the total percentage of body fat $z$-score.

\section{Results}

The baseline characteristics of the participants are presented in Table 1. There was no significant difference in mean age, Tanner stage, height $z$-score, BMI $z$-score, GRF-score, or diagnosis between the boys who were or were not treated with AP. The AP-treated boys had a higher disease severity score in comparison with the boys without AP treatment (4.7 for the AP-treated boys vs 4.0 for the boys without AP treatment respectively; $P=0.003)$. Of the boys treated with $\mathrm{AP}$, 53 were treated with risperidone at a mean dose of $1.6 \mathrm{mg} /$ day (s.D. 0.8 , range $0.5-4$ ) with mean treatment duration of 51.4 months (S.D. 28, range 16-126) and three were treated with pimozide at a mean dose of $1.7 \mathrm{mg} /$ day. Two-thirds of the AP-treated boys (37 out of 56) had used their AP medication for more than 3 years. The mean prolactin level in AP-treated boys was $18.9 \mathrm{ng} / \mathrm{ml}$ (s.D. 16, range 2.4-92.5); it was $28.08 \mathrm{ng} / \mathrm{ml}$ (s.D. 19.1 , range $14.6,92.5$ ) in the 27 boys with hyperprolactinemia and $10.38 \mathrm{ng} / \mathrm{ml}$ (s.D. 4.0 , range $2.4,17$ ) in the 29 boys without hyperprolactinemia. Four out of the 27 (15\%) hyperprolactinemic boys had possible prolactin-related sexual functioning disorders. The mean serum prolactin level was $8.0 \mathrm{ng} / \mathrm{ml}$ (S.D. 2.8, range 3.8-15.1) in the boys not treated with AP. The calcium intake was significantly lower in the AP-non-treated boys than in the AP-treated boys, but the latter had a significantly lower 25-hydroxyvitamin $\mathrm{D}_{3}$ level $(P=0.017)$. 25-Hydroxyvitamin $\mathrm{D}_{3}$ levels were deficient in eight $(14 \%)$ of the AP-treated boys and insufficient in 17 (30\%). Of the 47 boys not treated with AP, five (11\%) and six (13\%) had deficient and insufficient 25-hydroxyvitamin $\mathrm{D}_{3}$ levels respectively. Ten AP-treated boys (18\%) reported a history of a fracture compared with 12 boys not treated with AP (26\%; Table 1).

Table 2 Bone mineral density, body composition, and biochemical parameters of adolescent boys with autism spectrum disorders who were treated $(n=56)$ or not treated $(n=47)$ with antipsychotics (AP).

\begin{tabular}{|c|c|c|c|c|c|}
\hline & \multicolumn{3}{|c|}{ Treated with AP $(n=56)$} & \multicolumn{2}{|c|}{ Not treated with AP $(n=47)$} \\
\hline & $\begin{array}{l}\text { With hyperprolactinemia } \\
\qquad(n=27) \\
\text { Mean (s.D., range) }\end{array}$ & $\begin{array}{l}\text { Without hyperprolactinemia } \\
\qquad(n=29) \\
\text { Mean (S.D., range) }\end{array}$ & $P$ value $^{\mathrm{a}}$ & Mean (s.D., range) & $P$ value $^{\mathrm{b}}$ \\
\hline Lean tissue mass $z$-score & $-0.29(1.3 ;-2.24,2.31)$ & $-0.13(1.1 ;-1.92,2.75)$ & $0.69^{\mathrm{c}}$ & $-0.57(1.4 ;-0.87,0.15)$ & $0.67^{\mathrm{c}}$ \\
\hline Percentage of body fat $z$-score & $1.40(0.71 ;-0.26,2.95)$ & $1.10(0.83 ;-0.53,2.57)$ & $0.032^{c}$ & $1.06(0.90 ;-0.73,2.74)$ & $0.81^{\mathrm{c}}$ \\
\hline Lumbar spine BMD $z$-score & $-0.18(1.0 ;-2.36,1.69)$ & $0.15(0.78 ;-1.46,1.51)$ & $0.15^{\mathrm{c}}$ & $-0.026(1.17 ;-0.46,0.35)$ & $0.74^{\mathrm{c}}$ \\
\hline Total body BMD $z$-score & $0.038(1.16 ;-2.1,2.84)$ & $0.083(0.81 ;-1.24,2.24)$ & $0.79^{\mathrm{c}}$ & $-0.20(1.11 ;-0.67,0.14)$ & $0.80^{\mathrm{c}}$ \\
\hline Lumbar spine BMAD $z$-score & $-0.32(0.80 ;-2.49,0.75)$ & $0.12(0.77 ;-1.4,1.35)$ & $0.043^{c}$ & $-0.12(0.04 ;-0.36,0.30)$ & $0.33^{\mathrm{C}}$ \\
\hline P1NP $(\mu \mathrm{g} / \mathrm{l})$ & $462(286 ; 67,1033)$ & $589(313 ; 143,1111)$ & $0.060^{c}$ & $546.19(338.9 ; 61,1586)$ & $0.48^{\mathrm{C}}$ \\
\hline $\mathrm{BAP}(\mu \mathrm{g} / \mathrm{l})$ & $82.5(52.1 ; 10.7,173)$ & $98.4(60.3 ; 16,257)$ & $0.16^{\mathrm{c}}$ & $78.40(51.4 ; 14.3,241)$ & $0.062^{c}$ \\
\hline $\mathrm{CTx}(\mu \mathrm{g} / \mathrm{l})$ & $0.80(0.40 ; 0.23,1.51)$ & $0.99(0.39 ; 0.31,1.95)$ & $0.042^{c}$ & $1.11(0.52 ; 0.32,3.22)$ & $0.19^{\mathrm{c}}$ \\
\hline
\end{tabular}

BMD, bone mineral density; BMAD, bone mineral apparent density; P1NP, type 1 amino-terminal propeptide of procollagen; CTX, carboxyterminal crosslinking telopeptide of bone collagen; BAP, bone-specific alkaline phosphatase.

${ }^{a} P$ value compares boys treated with antipsychotics with and without hyperprolactinemia.

${ }^{\mathrm{b}} P$ value compares boys treated with antipsychotics with boys not treated with antipsychotics.

'Linear regression analysis corrected for BMI $z$-score, 25-hydroxyvitamin D, and average calcium daily intake. 


\section{Bone mass and body composition}

The mean $z$-scores of the lumbar spine, total body BMD, and the lumbar spine BMAD (volumetric BMD) for all boys $(n=103)$ were not significantly different from zero. The mean height $z$-score $(0.39, P<0.001)$ and total percentage of body fat $z$-score $(1.16, P<0.001)$ were significantly higher than normal and the mean lean tissue mass $z$-score $(-0.37, P<0.004)$ was significantly lower than normal.

Table 2 shows the differences between AP-treated boys with $(n=27)$ and without $(n=29)$ hyperprolactinemia and the differences between the boys who were or were not treated with AP. There were no significant differences in $z$-scores of the lumbar spine and total body $\mathrm{BMD}$, lumbar spine BMAD, lean tissue mass, and total percentage of body fat between the boys who were or were not treated with AP. Within the AP-treated boys, the boys with AP-induced hyperprolactinemia had a lower BMAD $z$-score $(P=0.043)$ and a higher total percentage of body fat $z$-score $(P=0.032)$ than did the AP-treated boys without hyperprolactinemia; there were no significant differences in mean $z$-scores of lumbar spine and total body BMD and lean tissue mass between the two groups of AP-treated boys. The BMD and body composition of the hyperprolactinemic boys with prolactin-related side effects (four out of 27) was not significantly different from the hyperprolactinemic boys without clinical prolactin-related signs.

Low lumbar spine BMAD (defined as a $z$-score of $\leq-2)$ was present in two out of the $27(7.4 \%)$ hyperprolactinemic AP-treated boys and in none of the boys with AP treatment without hyperprolactinemia and in none without AP treatment. The two boys with hyperprolactinemia and low BMD had no prolactin-related side effects, such as erectile dysfunction, gynecomastia, or loss of libido. Low lumbar spine BMD was present in three out of $27(11.1 \%)$ hyperprolactinemic AP-treated boys, of which one of these boys had a possible prolactin-related side effect; he reported a loss of libido and problems in the ability to have an orgasm, and low lumbar spine BMD was present in one out of $47(2.1 \%)$ boys without AP treatment and in none of the boys with AP treatment without hyperprolactinemia. Prolactin levels were positively associated with the mean total percentage of body fat $z$-score $(r=0.188, P=0.06)$, and the mean total percentage of body fat $z$-score was negatively associated with the GRF score $(r=-0.312, P=0.001)$.

\section{Biochemical bone parameters}

Table 2 shows that the levels of markers of bone resorption (CTx) and formation (BAP, P1NP) were not significantly different between the boys who were or were not treated with AP and were also normal compared with normative data (37). The AP-treated boys with hyperprolactinemia had significantly lower
CTx levels $(P=0.042)$ than the AP-treated boys without hyperprolactinemia. P1NP levels showed a trend toward being lower in the AP-treated boys compared with those without AP treatment $(P=0.06)$. BAP levels were not different between the two groups $(P=0.16)$. Prolactin levels were negatively correlated with CTx $(r=-0.20, P=0.048)$ and showed a trend toward negative correlation with P1NP levels $(r=-0.17, P=0.095)$. BAP was not associated with prolactin levels $(r=-0.05, P=0.60)$.

\section{Discussion}

\section{Bone mineral density}

Lumbar spine and total body BMD was found to be similar in boys with ASD/DBD with and without AP treatment and not significantly different from normal in this study. Importantly, we observed a diminished volumetric BMD of the lumbar spine for the group of boys with hyperprolactinemia compared with the boys with AP treatment without hyperprolactinemia. The volumetric BMD of the lumbar spine is a good predictor of future fractures (especially wrist and forearm fractures) $(45,46)$, and therefore, our findings suggest that AP-induced hyperprolactinemia may have a negative influence on BMD, especially of trabecular bone, such as of the lumbar vertebrae. Low BMD was present in $7 \%$ (volumetric lumbar spine BMD) and 11\% (areal lumbar spine BMD) respectively of the AP-treated hyperprolactinemic boys. Just one of these boys had possible clinical prolactin-related signs of hypogonadism; thus, clinicians should be aware that hyperprolactinemia may be associated with low BMD without alarming signs. In adults with AP-induced hyperprolactinemia, the effect on BMD and prevalence of fractures has been studied. Patients treated with these AP have a twofold risk in gaining an upper leg or hip fracture $(23,24)$. Patients with schizophrenia treated with AP-inducing hyperprolactinemia have significant diminished areal BMD compared with patients without hyperprolactinemia (47). However, there are conflicting results showing that lifestyle differences in schizophrenia may be the cause of the diminished BMD instead of the hyperprolactinemia (e.g. less exercise, nutritional alterations, and smoking) (48). In male children and adolescents with various psychiatric conditions, the effect of AP-induced hyperprolactinemia was investigated in one previous study (16). This study $(n=83$, mean age 12 years) reported a reduced BMD in the hyperprolactinemic boys. Comparisons with healthy controls were not made. However, both our study and the Calarge study had a cross-sectional design; therefore, it is not possible to draw any final conclusions about these results. Well-designed prospective studies are needed to assess the relative contributions of hyperprolactinemia, hypogonadotropic hypogonadism, the 
psychiatric condition, nutritional alterations, and other lifestyle changes on BMD and its clinical consequences.

There are two previous studies investigating the effect of ASD on bone status $(49,50)$. One study evaluated fracture prevalence and found this to be lower for patients with ASD, i.e. $12 \%$ for the patients with ASD vs $25 \%$ for the comparison group (follow-up for 30 years, $n=118$ ) (49), and the other study investigated bone cortical thickness in children with ASD (mean age 7 years, $n=75$ ) measured on a hand-wrist radiograph. When compared with normative data, the boys with ASD with a dairy-free diet had reduced bone cortical thickness (50). The patients of this study had a normal calcium intake and a fracture prevalence rate of about $20 \%$ (19\% for the ASD boys with hyperprolactinemia vs $21 \%$ for the ASD boys without hyperprolactinemia), which was also low, however, measured at a mean age of 23 years, compared with the normative data fracture rate of $37 \%$ (45). One explanation could be that boys with ASD have fewer organized outdoor sports activities, and their preferred leisure activities often involve an indoor setting, which would protect them from sustaining fractures (51).

\section{Biochemical bone markers}

Our study is the first to investigate bone markers in boys with hyperprolactinemia. The levels of bone resorption marker CTx were significantly lower and levels of bone formation marker P1NP tended to be lower in AP-treated boys with hyperprolactinemia than in AP-treated boys without hyperprolactinemia. This suggests that hyperprolactinemia may inhibit bone turnover, possibly by a direct negative effect on osteoblasts, or by a secondary effect through hyperprolactinemia-induced hypogonadotropic hypogonadism. We have reported before that the boys with hyperprolactinemia had significantly lower testosterone levels (52), which may have resulted in a decrease in bone turnover. Further research in this area is needed as bone turnover markers reflect the dynamic aspect of bone metabolism, and longitudinal data are needed to investigate the changes over time.

\section{Body composition}

We found, as expected, lean tissue mass to be lower and the percentage of mean total body fat to be higher in our total study population than in healthy controls, which may be due to disease-specific differences in lifestyle. As mentioned earlier, children or adolescents with ASD do not commonly participate in organized outdoor sports, preferring indoor leisure activities (51). The GRF score, a parameter of physical activity, was negatively associated with fat percentage. Lean tissue mass, which is mainly muscle mass, is highly related to bone mass in children and is important for the attainment of peak bone mass (45). It is reassuring from a clinical perspective that the psychiatric condition of ASD or infantile autism does not seem to lead to a higher fracture prevalence rate. Prolactin levels were positively associated with the total percentage of body fat $z$-scores. As the AP-treated boys were earlier found to have low, but still within the normal range, testosterone levels (52), the lower testosterone levels could explain in part the higher percentage of body fat in relation to the lean tissue mass in these boys because testosterone is an important mediator of gain in muscle mass in adolescence (53).

\section{5-Hydroxyvitamin D3}

25-Hydroxyvitamin D3 is a determinant of BMD in children and adolescents (54). Thirteen of the 113 boys (13\%) had deficient levels of 25-hydroxyvitamin D3 and $37(36 \%)$ insufficient levels; these are higher proportions than reported for Dutch young adult males without psychiatric disorders (4 and 22\% respectively) (55). This difference in 25-hydroxyvitamin D3 levels could be due to the disease-specific dietary restrictions and/or food selectivity (56), a lack of exposure to sunlight, given the preferred indoor activities of our ASD population, or seasonal variance (51).

\section{Limitations}

This study had some limitations that should be taken into account when interpreting the results and which should be addressed in future studies. As the study was observational and cross-sectional, we cannot establish causality and the power of the study was limited because of the relatively small sample size. Peak bone mass is usually attained between 18 and 23 years (45), and as our study participants were about 15 years old, bone growth, modeling, and remodeling were active, which makes it difficult to evaluate bone metabolism at one time point. Another potential limitation was the choice to measure BMD using DXA, instead of the more sensitive peripheral quantitative computed tomography. Although DXA is the clinical standard, trabecular bone, which is more vulnerable than cortical bone to hormonal abnormalities, such as hyperprolactinemia (57), cannot be distinguished from cortical bone by DXA, and thus early effects of hyperprolactinemia on BMD might have been missed (58). Even so, we did find significant differences in the lumbar spine BMAD, measured by DXA, between the AP-treated boys with and without hyperprolactinemia.

We further measured bone turnover markers, which reflect the dynamic aspect of bone metabolism; however, bone markers of bone turnover are difficult to interpret, especially in children and adolescents as they reflect both growth and bone modeling and remodeling. Longitudinal data are needed to investigate the changes over time and to establish a clinically important 
difference in these markers due to AP-induced hyperprolactinemia.

The boys with AP were more severely ill than the boys without AP, and we cannot exclude that severity of the illness had a negative effect on BMD. We could hypothesize that more severely ill patients had specific dietary restrictions and/or food selectivity and less physical exercise and this could have had a negative influence on their BMD. Another potential limitation is that the boys were allowed to use concomitant prolactin-elevating medication, such as melatonin and selective serotonin reuptake inhibitors, which could have influenced the prolactin levels and BMD (16). However, use of these medications was similar in both groups and probably did not confound our results. Moreover, the prolactin-elevating effect of this concomitant medication is considered to be relatively minor compared with that of the AP used (59).

\section{Conclusion}

This study suggests that AP-induced hyperprolactinemia in boys with ASD may have a negative influence on BMD. Further research including longitudinal studies is needed to disentangle the effects of psychiatric conditions (ASD or DBD) and the effects of AP treatment and hyperprolactinemia. The physician should evaluate the history of fractures, calcium intake, sun exposure, and exercise in boys with hyperprolactinemia due to AP treatment. Assessment of serum 25-hydroxyvitamin D3 and BMD measurement should be considered. If AP-induced hyperprolactinemia occurs, the AP should be reduced if this is clinically feasible. Prolactin levels should normalize within days dependent on the half-life (T1/2) of the drug (60). If this is not effective, a switch to an AP without prolactin-elevating qualities may be useful $(61,62)$.

\section{Declaration of interest}

Y Roke, D E Tenback, Y B de Rijke, A M Boot, and L G B A Quekel have no actual or potential conflict of interest. They did not participate in scientific advisory boards, did not receive an industrial grant support, and did not have stocks or options of pharmaceutical companies or patent royalties. $\mathrm{P} \mathrm{N}$ van Harten did not participate in scientific advisory boards, did not receive an industrial grant support, and did not have stocks or options of pharmaceutical companies or patent royalties. In the last year, he gave lectures at symposia sponsored by Lilly BV, Bristol Meyers Squibb, and Glaxo Smith Kline for which he received an honorarium. J K Buitelaar has been in the past 3 years a consultant to/member of advisory board of/and/or speaker for Janssen Cilag BV, Eli Lilly, Bristol-Myer Squibb, Shering Plough, UCB, Shire, Medice, and Servier. He is not an employee of any of these companies and not a stock shareholder of any of these companies. He has no other financial or material support, including expert testimony, patents, and royalties.

\section{Funding}

Funding of this study was provided by the following: i) Foundation 'De Open Ankh', Soesterberg, The Netherlands; ii) Foundation 'to support
VCVGZ', Arnhem, The Netherlands; and iii) Fund for Scientific Research of Sexuality, Amsterdam, The Netherlands. The study sponsors had no role in the study design, data collection, analysis and interpretation of the data, writing of the report, and the decision to submit the paper for publication.

\section{References}

1 McDougle C, Stigler K, Erickson C \& Posey D. Atypical antipsychotics in children and adolescents with autistic and other pervasive developmental disorders. Journal of Clinical Psychiatry 200869 15-20. (doi:10.4088/JCP.0508e15)

2 Aman M, McDougle C, Scahill L, Handen B, Arnold L, Johnson C, Stigler K, Bearss K, Butter E, Swiezy N et al. Medication and parent training in children with pervasive developmental disorders and serious behavior problems: results from a randomized clinical trial. Journal of the American Academy of Child and Adolescent Psychiatry 200948 1143-1154. (doi:10.1097/CHI.0b013e3181 bfd669)

3 Research Units on Pediatric Psychopharmacology Autism Network. Risperidone in children with autism and serious behavioral problems. New England Journal of Medicine 2002347 314-321. (doi:10.1056/NEJMoa013171)

4 Blankenship K, Erickson C, Stigler K, Posey DJ \& McDougle CJ. Aripiprazole for irritability associated with autistic disorder in children and adolescents aged 6-17 years. Pediatric Health 20104 375-381. (doi:10.2217/phe.10.45)

5 Wink L, Erickson C \& McDougle C. Pharmacologic treatment of behavioral symptoms associated with autism and other pervasive developmental disorders. Current Treatment Options in Neurology 201012 529-538. (doi:10.1007/s11940-010-0091-8)

6 Newcomer J. Second-generation (atypical) antipsychotics and metabolic effects: a comprehensive literature review. CNS Drugs 200519 1-93. (doi:10.2165/00023210-200519001-00001)

7 Correll C, Manu P, Olshanskiy V, Napolitano B, Kane J \& Malhotra A. Cardiometabolic risk of second-generation antipsychotic medications during first-time use in children and adolescents. Journal of the American Medical Association 200921 2302-2322.

8 Roke Y, van Harten P, Boot A \& Buitelaar J. Antipsychotic medication in children and adolescents: a descriptive review of the effects on prolactin level and associated side effects. Journal of Child and Adolescent Psychopharmacology 200919 403-414. (doi:10.1089/cap.2008.0120)

9 Houseknecht K, Robertson A, Zavadoski W, Gibbs E, Johnson D \& Rollema H. Acute effects of atypical antipsychotics on whole-body insulin resistance in rats: implications for adverse metabolic effects. Neuropsychopharmacology 200732 289-297. (doi:10.1038/sj.npp.1301209)

10 Schwetz V, Pieber T \& Obermayer-Pietsch B. Mechanisms in endocrinology: The endocrine role of the skeleton: background and clinical evidence. European Journal of Endocrinology 2012166 959-967. (doi:10.1530/EJE-12-0030)

11 Davies J, Evans B \& Gregory J. Bone mass acquisition in healthy children. Archives of Disease in Childhood 200590 373-378. (doi:10.1136/adc.2004.053553)

12 Taes Y, Lapauw B, Vanbillemont G, Bogaert V, De Bacquer D, Zmierczak $\mathrm{H}$ \& Goemaere S. Fat mass is negatively associated with cortical bone size in young healthy male siblings. Journal of Clinical Endocrinology and Metabolism 200994 2325-2331. (doi:10.1210/jc.2008-2501)

13 Seriwatanachai D, Thongchote K, Charoenphandhu N, Pandaranandaka J, Tudpor K, Teerapornpuntakit J, Suthiphongchai T \& Krishnamra N. Prolactin directly enhances bone turnover by raising osteoblast-expressed receptor activator of nuclear factor $\mathrm{\kappa B}$ ligand/osteoprotegerin ratio. Bone $2008 \mathbf{4 2}$ 535-546. (doi:10.1016/j.bone.2007.11.008)

14 Motyl K, Dick-de-Paula I, Maloney A, Lotinun S, Bornstein S, de Paula F, Baron R, Houseknecht K \& Rosen C. Trabecular bone 
loss after administration of the second-generation antipsychotic risperidone is independent of weight gain. Bone $2012 \mathbf{5 0}$ 490-498. (doi:10.1016/j.bone.2011.08.005)

15 Graham S, Howgate D, Anderson W, Howes C, Heliotis M, Mantalaris A, Tsiridis E \& Tsapakis E. Risk of osteoporosis and fracture incidence in patients on antipsychotic medication. Expert Opinion on Drug Safety 201110 575-602. (doi:10.1517/ 14740338.2011.560112)

16 Calarge C, Zimmerman B, Xie D, Kuperman S \& Schlechte J. A cross-sectional evaluation of the effect of risperidone and selective serotonine reuptake inhibitors on bone mineral density in boys. Journal of Clinical Psychiatry 20101 338-347. (doi:10.4088/JCP. $08 \mathrm{m04595gre)}$

17 Colao A, Di Somma C, Loche S, Di Sarno A, Klain M \& Pivonello R. Prolactinomas in adolescents: persistent bone loss after 2 years of prolactin normalization. Clinical Endocrinology 200052 319-327. (doi:10.1046/j.1365-2265.2000.00902.x)

18 Abraham G, Halbreich U, Friedman R \& Josiassen R. Bone mineral density and prolactin associations in patients with chronic schizophrenia. Schizophrenia Research 200359 17-18. (doi:10.1016/S0920-9964(01)00321-8)

19 Abraham G, Paing W, Kaminski J, Joseph A, Kohegyi E \& Josiassen R. Effects of elevated serum prolactin on bone mineral density and bone metabolism in female patients with schizophrenia: a prospective study. American Journal of Psychiatry 2003 160 1618-1620. (doi:10.1176/appi.ajp.160.9.1618)

20 Meaney A, Smith S, Howes O, O'Brien M, Murray R \& O'Keane V. Effects of long-term prolactin-raising antipsychotic medication on bone mineral density in patients with schizophrenia. British Journal of Psychiatry $2004 \mathbf{1 8 4}$ 503-508. (doi:10.1192/bjp. 184.6.503)

21 O'Keane V \& Meaney A. Antipsychotic drugs: a new risk factor for osteoporosis in young women with schizophrenia? Journal of Clinical Psychopharmacology 200525 26-31. (doi:10.1097/01. jcp.0000150223.31007.e0)

22 Becker A \& Epperson C. Female puberty: clinical implications for the use of prolactin-modulating psychotropics. Child and Adolescent Psychiatric Clinics of North America 200815 207-220. (doi:10. 1016/j.chc.2005.08.006)

23 Hugenholtz GW, Heerdink ER, van Staa TP et al. Risk of hip/femur fractures in patiens using antipsychotics. Bone 200537 864-870.

24 Howard L, Kirkwood G \& Leese M. Risk of hip fracture in patients with a history of schizophrenia. British Journal of Psychiatry 2007 190 129-134. (doi:10.1192/bjp.bp.106.023671)

25 Verhelst J \& Abs R. Hyperprolactinemia: pathophysiology and management. Treatments in Endocrinology 2003 2 23-32. (doi:10.2165/00024677-200302010-00003)

26 Fredriks A, van Buuren S, Burgmeijer R, Meulmeester J, Beuker R, Brugman E, Roede M, Verloove-Vanhorick S \& Wit J. Continuing positive secular growth change in The Netherlands 1955-1997. Pediatric Research 2000a 47 316-323. (doi:10.1203/00006450200003000-00006)

27 Fredriks A, van Buuren S, Wit J \& Verloove-Vanhorick S. Body index measurements in 1996-7 compared with 1980. Archives of Disease in Childhood $2000 b 82$ 107-112. (doi:10.1136/ adc.82.2.107)

28 Duke P, Litt I \& Gross R. Adolescents' self-assesment of sexual maturation. Pediatrics 198066 918-920.

29 Guy W. ECDEU Assessment Manual for Psychopharmacology, pp 217-222. Rockville, MD: U.S. Department of Health, Education, and Welfare, 1976.

30 Angus R, Sambrook P \& Pocock N. A simple method for assessing calcium intake in Caucasian women. Journal of the American Dietetic Association 198989 209-214.

31 Greendale G, Huang M. Wang Y. Finkelstein J. Danielson M \& Sternfeld B. Sport and home physical activity are independently associated with bone density. Medicine and Science in Sports and Exercise 200335 506-512. (doi:10.1249/01.MSS.0000056725. 64347.C9)

32 Kemper H, Twisk J \& van Mechelen W. A fifteen-year longitudinal study in young adults on the relation of physical activity and fitness with the development of the bone mass: the Amsterdam Growth and Health Longitudinal Study. Bone 200027 847-853. (doi:10.1016/S8756-3282(00)00397-5)

33 Bakker I, Twisk J, van Mechelen W, Roos J \& Kemper H. Ten-year longitudinal relationship between physical activity and lumbar bone mass in (young) adults. Journal of Bone and Mineral Research 200318 325-332. (doi:10.1359/jbmr.2003.18.2.325)

34 Amadori P, Dilberis C \& Marcolla A. All the studies on hyperprolactinemia should not forget to consider the possible presence of macroprolactinemia. European Journal of Endocrinology 2004150 93-94. (doi:10.1530/eje.0.1500093)

35 Emlinger M, Kühnel W \& Ranke M. Reference ranges for serum concentrations of lutotropin (LH), follitropin (FSH), estradiol $\left(\mathrm{E}_{2}\right)$, prolactin, progesterone, sex hormone binding globuline (SHBG), dehydroepiandrosterone sulfate (DHEAS), cortisol and ferritin in neonates, children and young adults. Clinical Chemistry and Laboratory Medicine 200240 1151-1160.

36 Van der Sluis I, de Ridder M. Boot A. Krenning E \& de Muinck Keizer-Schrama S. Reference data for bone density and body composition measured with dual energy X ray absorptiometry in white children and young adults. Archives of Disease in Childhood 200287 341-347. (doi:10.1136/adc.87.4.341)

37 Rauchenzauner M, Schmid A, Heinz-Erian P, Kapelari K, Falkensammer G, Griesmacher A, Finkenstedt G \& Högler W. Sex- and age-specific reference curves for serum markers of bone turnover in healthy children from 2 months to 18 years. Journal of Clinical Endocrinology and Metabolism 200792 443-449. (doi:10.1210/jc.2006-1706)

38 Allgrove J \& Shaw N. Calcium and Bone Disorders in Children and Adolescents. Vol 16 pp1-10. Basel: Karger, 2009.

39 Margulies L, Horlick M, Thornton J, Wang J, Ioannidou E \& Heymsfield S. Reproducibility of pediatric whole body bone and body composition measures by dual-energy X-ray absorptiometry using the GE Lunar Prodigy. Journal of Clinical Densitometry 20058 298-304. (doi:10.1385/JCD:8:3:298)

40 Kröger H, Kotaniemi A, Vainio P \& Alhava E. Bone densitometry of the spine and femur in children by dual-energy X-ray absorptiometry. Bone and Mineral 199217 75-85. (doi:10.1016/01696009(92)90712-M)

41 Boot A, Bouquet J, de Ridder M, Krenning E \& de Muinck KeizerSchrama S. Determinants of body composition measured by dualenergy X-ray absorptiometry in Dutch children and adolescents. American Journal of Clinical Nutrition 199766 232-238.

42 Atkins M, Burgess A, Bottomley C \& Riccio M. Chlorpromazine equivalents: a consensus of opinion for both clinical and research applications. Psychiatric Bulletin 199721 224-226. (doi:10.1192/pb.21.4.224)

43 Woods S. Chlorpromazine equivalent doses for the newer atypical antipsychotics. Journal of Clinical Psychiatry 200364 663-667. (doi:10.4088/JCP.v64n0607)

44 Lewiecki EM, Gordon CM, Baim S, Leonard MB, Bishop NJ, Bianchi M, Kalkwarf HJ, Langman CB, Plotkin H, Rauch F, Zemel BS, Binkley N, Bilezikian JP, Kendler DL, Hans DB \& Silverman S. International Society for Clinical Densitometry 2007 Adult and Pediatric Official Positions. Bone 200843 1115-1121. (doi:10.1016/j.bone.2008.08.106)

45 Boot A, de Ridder M, van der Sluis I, van Slobbe I, Krenning E \& Keizer-Schrama S. Peak bone mineral density, lean body mass and fractures. Bone 201046 336-341. (doi:10.1016/j.bone.2009. 10.003)

46 Jones G, Ma D \& Cameron F. Bone density interpretation and relevance in Caucasian children aged 9-17 years of age: insights from a population-based fracture study. Journal of Clinical Densitometry 20069 202-209. (doi:10.1016/j.jocd.2006.02.004)

47 Kishimoto T, Watanabe K, Shimada N, Makita K, Yagi G \& Kashima H. Antipsychotic-induced hyperprolactinemia inhibits the hypothalamo-pituitary-gonadal axis and reduces bone mineral density in male patients with schizophrenia. Journal of Clinical Psychiatry 200869 385-391. (doi:10.4088/JCP.v69n0307)

48 Sugawara N, Yasui-Furukori N, Fujii A, Saito M, Sato Y, Nakagami T, Tsuchimine S \& Kaneko S. No association 
between bone mass and prolactin levels among patients with schizophrenia. Human Psychopharmacology 201126 596-601. (doi:10.1002/hup.1250)

49 Mouridsen S, Rich B \& Isager T. Fractures in individuals with and without a history of infantile autism. A Danish register study based on hospital discharge diagnoses. Journal of Autism and Developmental Disorders 201242 619-624. (doi:10.1007/ s10803-011-1286-x)

50 Hediger ML, England LJ, Molloy CA, Yu KF, Manning-Courtney P \& Mills JL. Reduced bone cortical thickness in boys with autism or autism spectrum disorder. Journal of Autism and Developmental Disorders 200838 848-856. (doi:10.1007/s10803-007-0453-6)

51 Macdonald M, Esposito P \& Ulrich D. The physical activity patterns of children with autism. BMC Research Notes 201118 4-422.

52 Roke Y, van Harten P, Buitelaar J, Tenback D, de Rijke Y \& Boot A. Antipsychotic-induced hyperprolactinemia and testosterone levels in boys. Hormone Research in Paediatrics 201277 235-240. (doi:10.1159/000337910)

53 Herbst K, Anawalt B, Amory J, Matsumoto A \& Bremner W. The male contraceptive regimen of testosterone and levonorgestrel significantly increases lean mass in healthy young men in 4 weeks, but attenuates a decrease in fat mass induced by testosterone alone. Journal of Clinical Endocrinology and Metabolism 200888 1167-1173. (doi:10.1210/jc.2002-020918)

54 Pekkinen M, Viljakainen H, Saarnio E, Lamberg-Allardt C \& Mäkitie O. Vitamin D is a major determinant of bone minera density at school age. PLOS ONE 20127 1-7. (doi:10.1371/ journal.pone.0040090)

55 Boot A, Krenning E \& de Muinck Keizer-Schrama S. The relation between 25-hydroxyvitamin $\mathrm{D}$ with peak bone mineral density and body composition in healthy young adults. Journal of Pediatric Endocrinology $\mathcal{E}$ Metabolism 201124 355-360.
56 Kodak T \& Piazza C. Assessment and behavioral treatment of feeding and sleeping disorders in children with autism spectrum disorders. Child and Adolescent Psychiatric Clinics of North America 200817 887-905. (doi:10.1016/j.chc.2008.06.005)

57 Schlechte J, Walkner L \& Kathol M. A longitudinal analysis of premenopausal bone loss in healthy women and women with hyperprolactinemia. Journal of Clinical Endocrinology and Metabolism 199275 698-703. (doi:10.1210/jc.75.3.698)

58 Pitukcheewanont $\mathrm{P} \&$ Chen P. Bone density measurements in children and adolescents. Quantitative computed tomography versus dual-energy X-ray absorptiometry. Endocrinologist 200515 232-239. (doi:10.1097/01.ten.0000170853.99431.15)

59 Coker F \& Taylor D. Antidepressant-induced hyperprolactinaemia. CNS Drugs 201024 563-574. (doi:10.2165/11533140-000 000000-00000)

60 Turrone P, Kapur S, Seeman M \& Flint A. Elevation of prolactin levels by atypical antipsychotics. American Journal of Psychiatry 2002159 133-135. (doi:10.1176/appi.ajp.159.1.133)

61 Stevens J, Kymissis P \& Baker A. Elevated prolactin levels in male youths treated with risperidone and quetiapine. Journal of Child and Adolescent Psychopharmacology 200515 893-900. (doi:10.1089/ cap.2005.15.893)

62 Greenaway M \& Elbe D. Focus on aripiprazole: a review of its use in child and adolescent psychiatry. Journal of the Canadian Academy of Child and Adolescent Psychiatry 200918 250-260.

Received 13 June 2012

Revised version received 30 August 2012

Accepted 25 September 2012 